\title{
REGENT: A Framework for Realistic Generation of Network Traffic
}

\author{
Dinil Mon Divakaran, Kar Wai Fok, Li Ling Ko*, Yung Siang Liau, Vrizlynn L. L. Thing \\ Department of Cyber Security \& Intelligence, Institute for Infocomm Research (I²R), A*STAR, Singapore \\ 138632.
}

* Corresponding author. Tel.: 64082035; email: koll@i2r.a-star.edu.sg

Manuscript submitted September 4, 2015; accepted April 26, 2016.

doi: $10.17706 /$ ijcce.2016.5.6.430-440

\begin{abstract}
Network security research is highly dependent on traffic datasets. Fair and comprehensive analysis as well as performance evaluations of different solutions for problems such as detections of intrusions, anomalies and attacks, requires traffic traces that closely resemble data from operational network. Currently available datasets are either getting obsolete with time, or lacking important information such as ground truth of data and payloads in the traffic. While making little compromises, an alternate solution to this problem is to generate traffic data. However, care has to be taken that such a solution is capable to cope up with the changing characteristics of traffic; more generally, it should be flexible enough to generate traffic with specific characteristics as required by a user. In this work, we develop a framework for realistic generation of network traffic, called REGENT, which takes traffic models as input. In REGENT, different protocols generate real traffic independently, and based on the specific models (such as distribution for inter-arrival time between connections, distribution for connection size, etc.) provided by a user. We conduct experiments wherein REGENT takes protocol models as input, and generates real traffic as output. Using analysis, we show that the characteristics of the generated traffic (protocols) are close to the models specified as input.
\end{abstract}

Key words: Network traffic, protocols, security.

\section{Introduction}

As the penetration of the Internet grows, the impact of threats, attacks, intrusions, security breaches and cyber crimes in the cyberspace has serious consequences affecting large number of businesses and users. The network that revolutionized the way people communicate is increasingly becoming vulnerable to malicious activities that can lead to anything from small-scale and losses to catastrophes.

Over the years, the network research community has worked towards addressing the challenges of network security, and designed solutions to detect and mitigate different malicious activities. Many solutions model and analyze network traffic, for purposes such as classification of application protocols, detection and mitigation of DoS (denial-of-service) attacks, network anomaly detection, etc. Hence, network traffic is an important and unavoidable component of network security research.

Obtaining realistic and useful network traffic for evaluating solutions is a major concern among researchers. Solutions are often evaluated on different datasets, impeding useful performance comparison studies. While network scenarios can be simulated to generate data for analysis, often the simulated datasets are not realistic. Therefore, the first preference is to obtain real network traffic traces. However, 
accessing traffic traces is difficult due to privacy concerns-careful analysis of traffic can leak sensitive information of customers and employees. Hence, most network traces available today are heavily anonymized and carry no payloads, in effect reducing the utility of the datasets. Besides, the datasets are not labeled with ground truth (by the application protocol, the file-types carried in connections, characteristics such as normal or anomalous, type of attacks, etc.). For example, CAIDA has been providing real Internet data for research for many years; but the traces neither contain payload nor are labeled [1].

Another obstacle is that collected network traces may not contain traffic characteristics that are interesting for particular research problems. For example, for a convincing evaluation of a new anomaly detection solution, the data should contain different types of anomalies mixed with normal traffic. Similarly, solutions developed specifically for Cloud datacenter networks should require traffic data that represent workflow in datacenters; here the dependence is on both the data and the topology (datacenters usually have well-defined network topologies). Though easy to demand, such requirements are challenging to meet.

To assist research on network security, we aim to develop a framework that can generate realistic traffic. We argue that an important property of a traffic generation framework should be flexibility to adapt to changing traffic models. To be flexible, a framework should take models, such as HTTP and SMTP models, as input. This is a deviation from the static traffic generators, which take just one, possibly the latest, model of traffic, and generate traffic accordingly. As a result, static generators cannot adapt to changing traffic characteristics. HTTP is a good example of changing models - HTTP characteristics have undergone significant changes due to changes in content type access (from text to images to videos), connecting technologies (mobile phones, for example), data-distribution solutions (content delivery networks, social networks, etc.) [2], [3]. The framework should also allow for easy configuration of the network topology, link capacities and delays, as well as the number of end-hosts participating in traffic generation.

In this paper, we present REGENT, a framework we developed for the realistic generation of network traffic. The most important aspect of the framework is its flexibility - it can take any given traffic model as input and generate traffic that closely follows the specified model. Hence, the realism of the generated traffic is dependent only on the input models. Observe that models for real traffic is usually easy to obtain, primarily because such abstractions do not capture sensitive information of the users in the network (as an example, see [4]). The contents in the traffic, the IP addresses accessed, the time and duration of specific connections, etc. are not captured with models; therefore, concern regarding data privacy is minimized. Besides, due to its flexibility, REGENT can be used to generate different traffic datasets resembling different organizations. Not all organizations have the same traffic model; for example, Internet traffic of banking organizations is characteristically different from those of universities. Our solution provides a mechanism to take the specific models of interest, and generate traffic following the models. Finally, as network traffic is generated by the framework, naturally REGENT also provides ground truth of the data.

In the next section, we discuss on related works. In Section 3, we list the properties desired in a traffic generation framework. We develop our framework for traffic generation REGENT in Section 4. In Section 5, we define a set of traffic models and generate data using REGENT; we also validate that the traffic thus generated by the framework fits the input model distributions.

\section{Related Works}

Network simulators are capable of generating network traffic. Among the simulators, ns-2 [5] is a widely used discrete event simulator where traffic-generating agents are defined following specific in-built models. However, the models are mostly for lower layer protocols of the TCP/IP stack. Also, ns-2 does not generate meaningful contents, and does not provide a way to control the content types carried by the application protocols. The relatively new ns-3 [6], [7] was developed to succeed ns-2; and as the platform is young and 
not complete, researchers have been developing new implementations to support traffic generation. For example, ref. [8] presents an implementation specifically for HTTP traffic generator in ns-3. Similarly, Ammar, Begin and Guerin-Lassous [9] developed a tool to generate traffic in ns-3. However, their generation is based on a specific model, namely the Poisson Pareto Burst Process, which is embedded in ns-3. Therefore, only the parameters of this model are tunable by a user; other models cannot be given as input.

To evaluate various DPI (deep packet inspection) tools, Bujlow, Carela-Español and Barlet-Ros used a testbed to generate real network traffic [10]. They generated traffic for a comprehensive set of widely used application protocols, for example, BitTorrent, Skype, Dropbox file sharing, web browsing, etc. The authors used available statistics to decide on various parameters of the testbed design, for example the number of Windows and Linux clients. However, as far as we understand, various statistics of the application protocols, such as inter-protocol distribution, flow-rate distribution per application, etc. were not considered. The testbed topology also presents a limitation, as it is simple and static with only minor variations possible.

A profile-based method to generate benchmark datasets is developed in [4]. The authors define two classes of profiles, one for attack scenarios and the other for encapsulating normal traffic behavior. The profiles for normal traffic are captured using real network activity of their research centre. These profiles are then used to generate traffic on a testbed of fixed topology. While we follow a similar approach, using profiles for generating traffic, REGENT's network topology is flexible and reconfigurable to fit any scenario, be it an enterprise network topology or a datacenter network topology.

Lastly, we also note that there are some network traces that are available online for the network research community. As mentioned earlier, CAIDA [1] has been providing real traces for some time now. Anonymised packet-header traces are also made available by LBNL [11]; these were collected during 2004-2005. We refer to [4] for a discussion on many of the publicly available datasets. We also note that NETRESEC has compiled a list of pcap files available on the Internet [12].

\section{Properties of a Traffic Generation Framework}

In this section, we list down the properties that are required in a framework for generating realistic network traffic data.

1) The traffic generation framework should be flexible to accept different models of traffic, both at application level (say, HTTP, SMTP, FTP, DNS, etc.) and at behavioral level (normal or malicious). The generated traffic should follow the specified models closely, and the data should be captured at packet level in standard pcap format.

2) The network topology should be reconfigurable based on the scenario specified by the user. This will allow users to generate data for wide varieties of scenarios, ranging from simple dumbbell topology to a datacenter network topology. With these two properties, it would be possible to generate a specific workflow for, say, a hierarchical (say, three-tier) datacenter network architecture using the framework.

3) The application protocols should carry meaningful contents; and the framework should allow users to specify the contents for data-transfer protocols. For example, consider a scenario where a researcher wants to experiment with a HTTP content-type classifier (ref. [13] is an example); for this, the researcher might need the framework to use the contents that she provides. In addition, distributions for content sizes and types should also be allowed to be specified as input where it is meaningful. For example, a user of the framework might specify the distributions of object sizes and object types for HTTP protocol; the user may also configure the framework to use the contents that she provides.

4) Ground truth of data, be it at the application or behavioral level, should be made available with the data. This should include labels for the application protocol and the content(s) carried in each connection ${ }^{1}$.

\footnotetext{
1 We use the terms flow and connection interchangeably.
} 


\section{REGENT: Design and Implementation}

In this section, we describe the design and implementation of our framework REGENT. The framework currently generates only normal traffic; in future, we will be extending it to generate attack traffic.

\subsection{End-Hosts and Network}

For generating real traffic, we need to either emulate or simulate two important entities: (i) end-hosts for sending as well as receiving traffic, and (ii) network for transporting traffic. The end-hosts and network should have a general representation to fit most common specifications expected from users.

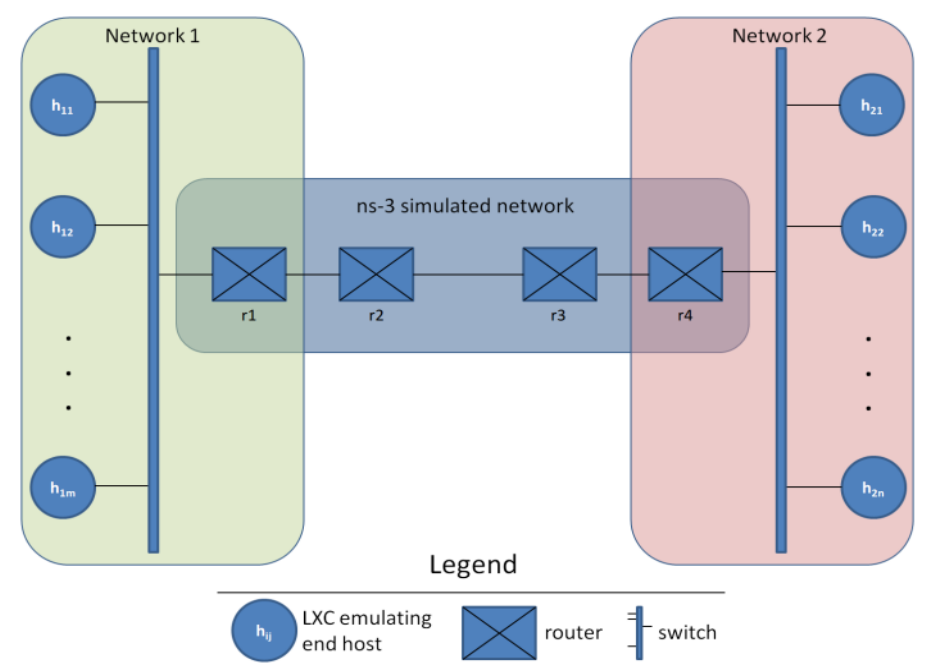

Fig. 1. Illustration of a scenario in the REGENT framework.

Testbeds usually employ machines to emulate end-host behaviors. This is not a scalable solution-even emulating a few tens of end-hosts incurs significant space and cost, much less hundreds to thousands of end-hosts to reflect the actual network. In this context, virtual machines come as a better option. Taking one more step, we realize that the end-hosts that need to be emulated can be a trimmed down version of a real user machine, as all that is expected from an end-host is the generation of traffic using a specific protocol. Therefore, it suffices that end-host executes just one single agent, where an agent uses a real implementation of a protocol. An example for an agent is an HTTP server. This led us to employ the lightweight virtualized environment, namely LXC, Linux Containers, to emulate the end-host [14].

Moving to the network representation, using a physical network for the traffic generation framework gives the advantage of emulating various router functions such as packet processing, scheduling and queuing, as closely as possible. But this is impractical, because the physical network testbed is limited in size, and smaller by orders in magnitude in comparison to the core network of the Internet. This makes it extremely difficult to emulate the core Internet behavior by using a testbed of just a handful of network elements (routers, switches and links). Besides, a physical network testbed is static in nature, limiting the possibilities of realizing various topologies (from simple dumbbell topology to AS topology to datacenter network topology). Considering these points, we decided to simulate the network (routers and links); for this purpose, we use ns-3 [6]. With ns-3, REGENT can deploy any network topology; besides, it has the capability to add or modify router functionalities and configure realistic link capacities and delays.

Fig. 1 illustrates the representation of a scenario in the framework. There are two networks of hosts, each host being emulated by a single LXC. While one network represents an enterprise or a university network, the other one is a logical network representing servers, clients and peers. These two networks of hosts are connected using the ns-3 simulator. The simple dumbbell topology is shown in this scenario. 


\subsection{Traffic Models and Contents}

As mentioned, REGENT takes models as inputs. A model can be specified for each relevant protocol characteristic. Below, we specify some important parameters that form input model specifications.

- Inter-protocol distribution: In a given scenario, traffic consisting of, say, $n$ different protocols need to be generated. To allow for different proportions, a user can specify the share of each protocol.

- Inter-arrival time distribution for flows: For each protocol, a user can specify the distribution of arrival times between flows (connections). For example, one can specify an Exponential distribution for inter-arrival times between HTTP flows. We highlight that, the flexibility to specify any distribution for different traffic characteristics, gives the user the capability to generate traffic closely following the scenario of interest. Along with the distribution, the parameters of the distribution are also specified.

- Content-size distribution: The distribution of the size of the contents carried by the connections and their parameters are given as input for data-transfer protocols such as HTTP, SMTP and FTP. The details of specification vary with protocols, and are given in the next section.

- Contents-types and contents: REGENT also allows users to specify the distribution of content types for protocols. For example, the distribution of the object types-text, HTML, PDF, JPEG, PNG, encrypted, binaries, archives, etc.- can be specified for data-transfer protocols like HTTP. A user can also provide contents that the protocol need to carry, by inputting the specific path of the directory containing the contents. If no content is specified, the framework uses the contents statically stored in the database. These stored contents are files retrieved from a Linux system, and are of various types such as text, HTML, image files in different formats, PDF, files encrypted using different algorithms, archives and compressed files, and binary executables. Files are selected based on the input size distribution specified as the model.

\subsection{Protocol Model}

The design of our framework allows protocols to be added and implemented in a modular way. REGENT currently has five application protocols implemented - HTTP, SCP/SSH, FTP, SMTP and DNS. The first four protocols were implemented using widely available Python libraries. A container can be configured to work as either a server or client, depending on the input scenario. For generating DNS traffic in a scenario, a DNS server is installed on a separate container before any traffic is generated. The DNS server provides primary DNS services to the rest of the containers whenever they perform DNS queries. With the DNS server in place, all containers are now associated with one or more domains, and whenever a container connects to another, a DNS query is initiated to which the DNS server responds with the IP address. The DNS traffic thus generated is captured and stored in the pcap file.

Models are taken as input for the four protocols - HTTP SCP, FTP and SMTP. We describe them below.

An HTTP request is a connection from a client to a web page. Associated with each request are a main object, and a number of inline objects. Based on this, the important parameters that our framework takes as input for modeling HTTP are [3]:

- Distribution of main object size

- Distribution of number of inline objects for each request

- Distribution of inline object size

- Distribution of inter-arrival time between web-page requests (reading time)

Our framework allows users to specify parameters to define a HTTP model. The implementation allows for named parameters and variable number of arguments as input, so that users need only provide necessary parameters to define distributions. For example, an Exponential distribution is realized using the mean (or rate) parameter, and a Pareto distribution is realized using scale and shape parameters. 
For FTP, SMTP and SCP, we allow the user to specify distributions for both the inter-arrival time between the connections and size of connections. For each of these protocols, the ratio of its contribution to that of HTTP, in number of connections, is also given as input.

- Parameters for SMTP:

- Distribution of attachment size

- Distribution of inter-arrival time between emails

- Parameters for SCP:

- Distribution of file size

- Distribution of inter-arrival time between connections

- Parameters for FTP:

- Distribution of session size, in number of connections per session

- Distribution of inter-arrival time between sessions

- Distribution of file size

- Distribution of inter-arrival time between connections in the same session

REGENT can also take complex distributions as input. For example, the attachment sizes of SMTP can be modeled using two distributions, one for small-size attachments, and another for large-size attachments. Similarly, FTP can have a distribution for short commands, and another for file transfers.

In response to each file-transfer request for any protocol, REGENT chooses a file whose size falls within a fixed percentage of the requested size. Currently, we do not discriminate between file types except when choosing HTTP's main object, where we will select only HTML files (as it is commonly the main object).

\subsection{Traffic Generation}

As mentioned, the most important feature of the REGENT framework is its flexibility in taking models as input. To make this possible, for each application at a container, REGENT has an agent that generates traffic. The agent is defined based on the protocol (e.g. HTTP), and can be a client or a server depending on the input scenario. The agent is responsible for generating traffic based on the model specified. The agents for different protocols execute independently.

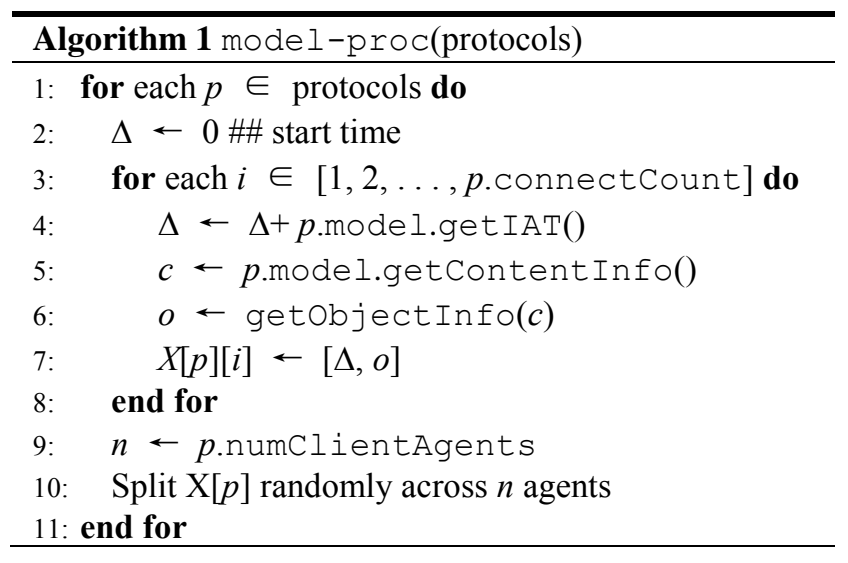

While the models for different protocols are independent of each other, the traffic generated by the different agents has to interleave to generate a mix of real traffic. As generating traffic using different protocols and models in a single pass is complicated, we split the task into two stages. We design the model-based traffic generation as follows. In the first stage, and before traffic generation, the models for the individual protocols as well as for the overall traffic (such as proportion of each protocol) will be taken as input by the model-proc component of the traffic generator, to produce a meta-file for each container. After this phase, the second phase begins wherein the agents generate traffic. 
A meta-file, in general, gives the following key information to a container:

- The application protocol to be used; this defines the agent at the container. For example, the agent might be an HTTP client.

- The number of connections with this container as the end-host. Details on each connection is provided:

- The time to generate each connection.

- If present, the information (path) of the file to be used for the connection.

- The peer (client/server) the agent is expected to connect to, for the specified connection.

Algorithm gives the high-level steps for model-proc. We shall explain using the HTTP protocol; i.e. $p=$ HTTP. The number of HTTP connections to be generated is HTTP.connectCount. The input model for HTTP provides the distributions and their parameters for (i) the inter-arrival time (IAT) between connections (requests), and (ii) the content size in a HTTP reply. Content-type distributions are also supported. These information are stored in HTTP.model, which can be retrieved using getIAT and getContentInfo functions. Sampling from the inter-arrival time distribution, we get the time between two HTTP connection requests; the absolute time of a connection initiation $\Delta$ is updated with this time (line 4:). Similarly, the content model has distribution for the object size, sampling from which we obtain the sizes of the objects in HTTP replies. Going further, as mentioned previously, the content type distribution can also be specified. This set of information is used to create the instance $o$ in line 6:. Depending on whether multiple objects are present in a request, $o$ holds one or more tuples. Each tuple contains the size and the path of the object (a file). Finally, $X$ holds details for all connections of a protocol $p$ in $X[p]$. The number of client agents to be used is given as input (HTTP.numClientAgents) and the connection information generated is randomly spread across the agents in the containers. Algorithm is used to create all the required information for connections of all protocols that transfers files (HTTP, SMTP, SCP and FTP).

\section{Validation}

REGENT generates network traffic across multiple containers, taking as input models for the protocols. The number of containers spawned is proportional to the number end-hosts modeled. As the number of containers increase, the system needs to cope up with increasing number of connection requests. Eventually, depending on the system configuration, this would affect the inter-arrival times between connections (and sessions). Similarly, file sizes might not always match the sample values from the distributions. In this section, we validate the generated traffic, to check if it follows the input models closely.

Table 1. HTTP Model Specification

\begin{tabular}{|l|l|}
\hline Parameter & Distribution and values \\
\hline \hline Main object size & $\begin{array}{l}\text { Weibull (shape }=0.81, \text { scale }= \\
28,000)\end{array}$ \\
\hline $\begin{array}{l}\text { Number of inline } \\
\text { objects per request }\end{array}$ & Exponential (mean $=0.30)$ \\
\hline Inline object size & Log-normal $(\mu=9.18, \sigma=1.25)$ \\
\hline $\begin{array}{l}\text { IAT (inter-arrival time) } \\
\text { between web-page } \\
\text { requests }\end{array}$ & Log-normal $(\mu=-0.50, \sigma=2.77)$ \\
\hline
\end{tabular}

Table 2. SMTP Model Specification

\begin{tabular}{|l|l|}
\hline Parameter & Distribution and values \\
\hline \hline File size & lower 80\%: Log-normal $(\mu=10.0, \sigma=$ \\
& $1.46)$ \\
& upper 80\%: Log-normal $(\mu=8.50, \sigma=$ \\
& $1.58)$ \\
\hline IAT between mails & Exponential $(\mu=352)$ \\
\hline
\end{tabular}


Table 3. SCP Model Specification

\begin{tabular}{|l|l|}
\hline Parameter & Distribution and values \\
\hline \hline File size & Exponential (mean $=89,800)$ \\
\hline $\begin{array}{l}\text { IAT between } \\
\text { connections }\end{array}$ & Exponential $(\mu=1,410)$ \\
\hline
\end{tabular}

Table 4. FTP Model Specification

\begin{tabular}{|l|l|}
\hline Parameter & Distribution and values \\
\hline \hline Session size & Log-normal $(\mu=9.17, \sigma=1.25)$ \\
\hline IAT between sessions & Exponential $(\mu=282)$ \\
\hline Connection size & Log-normal $(\mu=9.17, \sigma=1.25)$ \\
\hline $\begin{array}{l}\text { IAT between } \\
\text { connections in same } \\
\text { session }\end{array}$ & $\begin{array}{l}\text { short commands: exponential }(\mu= \\
\text { file transfers: log-normal }(\mu=3.27, \sigma \\
=2.16)\end{array}$ \\
\hline
\end{tabular}

In our experiment setup, we used the simple dumbbell topology illustrated in Fig. 1. The two networks contain 20 hosts each. Connections are made from hosts in the first network to hosts in the second network, as in a bipartite graph. For each source host and connection, the destination host in the second network is selected at random. We simulated more than 20,000 connections between the two networks. Link capacities and delays of all hosts are set to $1 \mathrm{Gbps}$ and $5 \mathrm{~ms}$, respectively. The bottleneck link of the network is set to a capacity of $10 \mathrm{Gbps}$ and delay of 10ms. We tested REGENT on a six-core 64-bit desktop computer, which runs on Intel Xeon CPU W3690 at a frequency of $3.47 \mathrm{GHz}$ and has 12GB RAM. The scenario used in our experiment is defined using the input models for the four protocols - HTTP, SMTP, SCP and FTP specified in Tables 1-4. Though DNS traffic is also generated in this experiment, it follows no model; DNS traffic is generated as and when the containers perform lookups.

The traffic generated by our framework REGENT for the above setup is captured and available at http://csi.i2r.a-star.edu.sg/nw-sec/traffic/.

We validate the generated traffic against the input models by applying the Kolmogorov-Smirnov (KS) test on object size and inter-arrival time distributions of each protocol. The KS test measures the goodness-of-fit to decide if a given sample set comes from a reference distribution. The test takes the samples and reference distribution function as inputs, and outputs a D-statistic and a p-value. The D-statistic is computed as the supremum of the absolute difference between the empirical distribution function (EDF) and the reference cumulative distribution function (CDF). Hence, smaller D-statistics signify better fit between the distributions. Under the null hypothesis that samples are generated from the reference distribution, the p-value measures of the probability of extremeness of the D-statistic. The lower the p-value, the higher the chances of extremeness. Therefore, larger p-values give more weight to the null hypothesis, and likewise smaller p-values increasingly discredit the null hypothesis. In statistical tests, we often fix a significance level $\alpha$ at 1,5 or $10 \%$, and accept the null hypothesis if the p-value is greater than $\alpha$, and vice-versa.

Besides performing the KS test, we also compare the EDF of the inter-arrival times and file/object sizes against their respective input model CDFs. Each EDF should match its CDF closely. In addition, we sketch the $95 \%$ confidence intervals of the EDF to verify that the input CDFs fall within those intervals.

Table 5 and Table 6 summarize the D-statistic and p-values from the KS test. Table 5 reports on the inter-arrival times of HTTP's web-page requests, SMTP's mails, SCP's transfer requests, and FTP's sessions. The last column reports the total sessions or requests for each protocol. Table 6 reports results for size distributions of HTTP's main objects, HTTP's inline objects, SMTP's attachments, SCP's files, and FTP's connections. The last column reports the total objects/attachments/files. 
Table 5. Kolmogorov-Smirnov Test on Inter-arrival Times

\begin{tabular}{cccc}
\hline \hline Protocol & D-statistic & p-value & No. sessions \\
\hline HTTP & 0.022 & $1.62 \mathrm{e}-7$ & 16,715 \\
SMTP & 0.023 & 0.355 & 1,646 \\
SCP & 0.063 & 0.076 & 408 \\
FTP & 0.016 & 0.689 & 2,056 \\
\hline \hline
\end{tabular}

Table 6. Kolmogorov-Smirnov Test on File-Size Distributions

\begin{tabular}{cccc}
\hline \hline Protocol & D-statistic & p-value & No. files \\
\hline HTTP (main) & 0.057 & $7.47 \mathrm{e}-49$ & 17,465 \\
HTTP (inline) & 0.022 & 0.593 & 3,398 \\
SMTP & 0.018 & 0.653 & 1,680 \\
SCP & 0.033 & 0.737 & 420 \\
FTP & 0.036 & $4.29 \mathrm{e}-5$ & 4,109 \\
\hline \hline
\end{tabular}

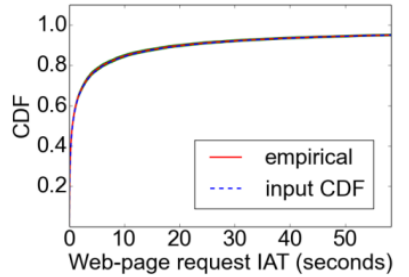

(a) HTTP

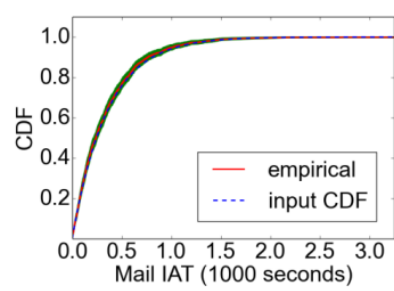

(b) SMTP

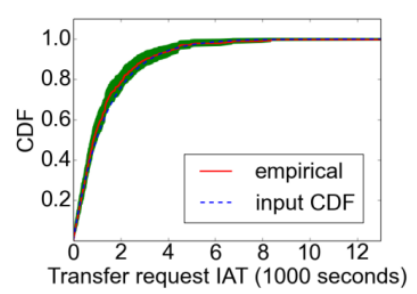

(c) SCP

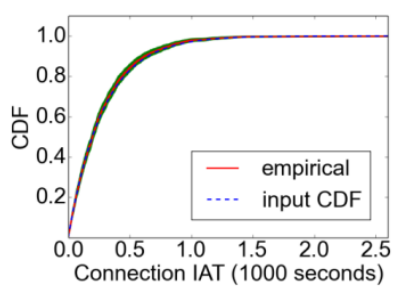

(d) FTP

Fig. 2. CDFs of inter-arrival times, for different protocols, with $95 \%$ confidence intervals shaded in green.

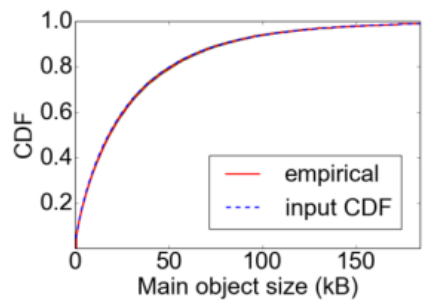

(a) HTTP (main object);

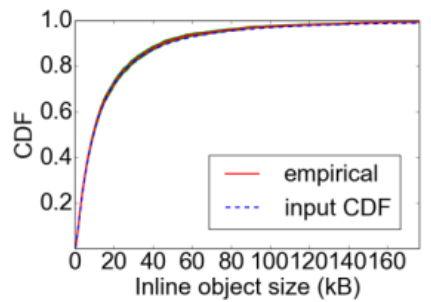

(b) HTTP (inline object);

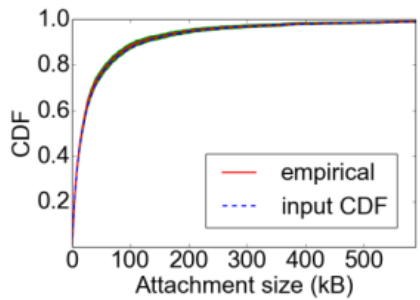

(c) SMTP

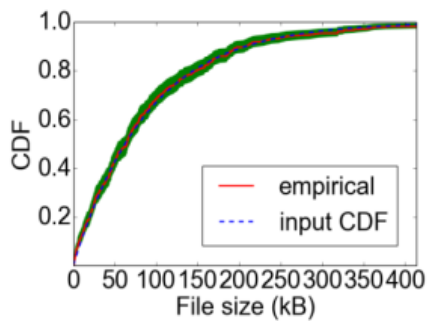

(d) SCP;

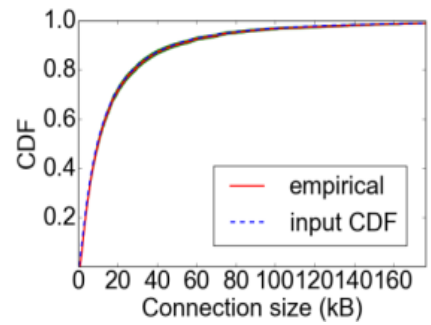

(e) FTP

Fig. 3. CDF of file sizes with $95 \%$ confidence intervals shaded in green.

Using a significance level of $\alpha=5 \%$, the KS test results suggest to accept the input CDF and observed distributions as the same for almost all distributions. The only three distributions with low p-values are for HTTP's main object size, FTP's connection size, and HTTP's IAT between web-page requests. The low p-values are counter-intuitive given the low D-statistics. We observe from the last columns of Table 5 and Table 6 that these distributions have large sample size, suggesting that the low p-values are an artifact of a large number of samples [15]. To support this claim, we compare the EDFs with their respective input CDFs.

Fig. 2 shows the graphs of inter-arrival times from Table 5, and Fig. 3 shows the graphs of file/object sizes 
from Table 6. All graphs show a good fit between the input CDFs and the EDFs. In particular, the input CDFs fall within the $95 \%$ confidence intervals of the EDFs. These results support that the traffic generated by REGENT fits the model specifications well for all inter-arrival times and file/object size distributions.

\section{Conclusion}

Network research, and particularly, research on network security is highly dependent on traffic data. In this work, we presented REGENT, the framework we developed for realistic generation of network traffic. REGENT is flexible as it can take traffic (protocol) models as input. As demonstration, we conducted experiments wherein models were defined and fed as input to REGENT, and consequently it generated real traffic for protocols as output. Using KS test, we showed that the traffic generated by the framework indeed follows the input models closely.

The REGENT framework currently supports five application protocols; as the design is modular, support for more protocols can be added in future. We plan to extend REGENT to generate attack traffic, again based on models for specific attacks. After which, the traffic generated by the framework can be used to evaluate various security solutions, such as those developed for detections of DDoS attacks, intrusions and malwares.

\section{References}

[1] CAIDA: Center for Applied Internet Data Analysis. From http://www.caida.org

[2] Ihm, S., \& Pai, V. S. (2011) Towards understanding modern web traffic. Proceedings of the 2011 ACM SIGCOMM Conference on Internet Measurement (pp. 295-308).

[3] Rastin, P., Magyari, Z., \& Tran-Gia, P. (2012). An HTTP web traffic model based on the top one million visited web pages. Proceedings of 8th EURO-NGI Conference on IEEE Next Generation Internet.

[4] Shiravi, A., Hadi-Shiravi, M. T., \& Ghorbani, A. A. (2012). Toward developing a systematic approach to generate benchmark datasets for intrusion detection. Computers \& Security, 31(3), 357-374.

[5] The Network Simulator NS-2. From http://www.isi.edu/nsnam/ns/

[6] The ns-3 network simulator. From http://www.nsnam.org

[7] Riley, G. F., \& Henderson, T. R. (2010). The ns-3 network simulator. Modeling and Tools for Network Simulation, Springer Berlin Heidelberg, 15-34.

[8] Cheng, Y., Çetinkaya, E. K., \& Sterbenz, J. P. (2013). Transactional traffic generator implementation in ns-3. Proceedings of the 6th International ICST Conference on Simulation Tools and Techniques (pp. 182-189).

[9] Ammar, D., Begin, T., \& Guerin-Lassous, I. (March 2011). A new tool for generating realistic internet traffic in NS-3. Proceedings of the 4th International ICST Conference on Simulation Tools and Techniques (pp. 81-83).

[10] Bujlow, T., Carela-Español, V., \& Barlet-Ros, P. (2014). Extended independent comparison of popular deep packet inspection (DPI) tools for traffic classification. Universitat Politècnica de Catalunya.

[11] LBNL/ICSI Enterprise Tracing Project, Lawrence Berkeley National Laboratory and ICSI. From http://www.icir.org/enterprise-tracing

[12] NETRESEC: Publicly available PCAP files. From http://www.netresec.com/?page=PcapFiles

[13] Khakpour, A. R., \& Liu, A. X. (2013). An information-theoretical approach to high-speed flow nature identification. IEEE/ACM Transactions on Networking, 21(4), 1076-1089.

[14] Linux containers. From https://linuxcontainers.org

[15] Lin, M., Lucas Jr., H. C., \& Shmueli, G. (2013). Research commentary-too big to fail: Large samples and the p-value problem. Information Systems Research, 24(4), 906-917. 


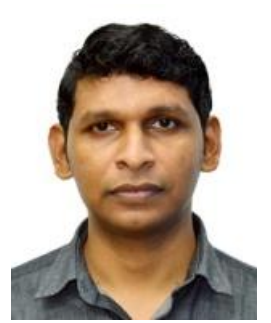

Dinil Mon Divakaran is a scientist at the Cyber Security \& Intelligence Department in the Institute for Infocomm Research in Singapore. Prior to this, for two years he was a research fellow at the Department of Electrical and Computer Engineering in the National University of Singapore (NUS). He has also worked as an assistant professor in the School of Computing and Electrical Engineering at the Indian Institute of Technology (IIT) Mandi. He carried out his Ph.D. degree at the INRIA team in ENS Lyon, France. He holds a master degree from IIT Madras, India. His research interests and experiences are in the areas of network security and QoS delivery. His research works revolve around applications of probabilistic models, game theory, queuing theory and machine learning, as well as the study of optimization problems and design of heuristics, all in the broad area of computer networks. He is a senior member of the IEEE.

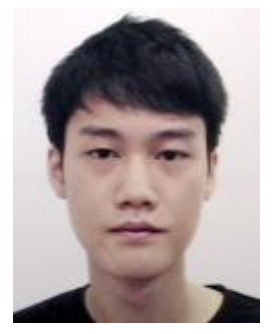

Kar Wai Fok received the B.Eng. degree in computer engineering from the National University of Singapore in 2015. He is currently a research engineer with the Cyber Security and Intelligence Department at the Institute for Infocomm Research, Singapore. His research interests include cyber security and network security.

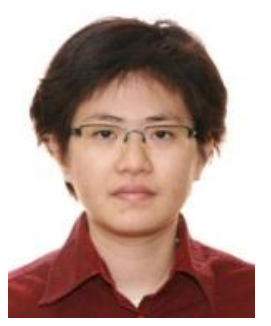

Li Ling Ko is a research engineer at the Cyber Security \& Intelligence department in the Institute for Infocomm Research, Singapore. She received her B.Eng. degree in computer engineering and the M.Sc. degree in mathematics from National University of Singapore, in 2008 and 2013 respectively. Her research interests include cyber security, security intelligence and analytics.

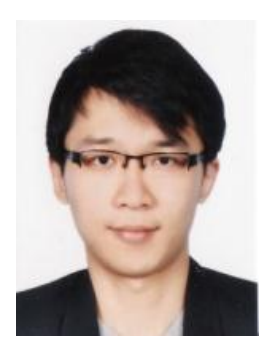

Yung Siang Liau received the B.Eng. degree in electrical engineering from the National University of Singapore, Singapore in 2012. He is currently a research engineer with the Cyber Security and Intelligence Department at the Institute for Infocomm Research, Singapore. His current research interests include security intelligence and machine learning.

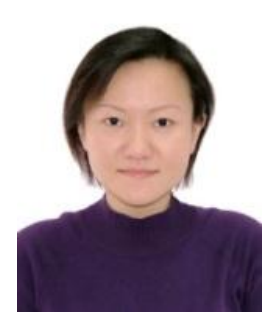

Vrizlynn L. L. Thing leads the Cyber Security \& Intelligence (CSI) R\&D Department at the Institute for Infocomm Research, A*STAR, Singapore. The department focuses on digital forensics, cybercrime analysis, cyber security \& intelligence, and mobile security research and technology development. She is also an adjunct associate professor at the National University of Singapore (School of Computing), and the Singapore Management University (School of Information Systems). She has over 13 years of security and forensics R\&D experience with in-depth expertise in various aspects of cyber security, forensics \& intelligence. Her research draws on her multidisciplinary background in computer science (Ph.D. from Imperial College London, United Kingdom), and electrical, electronics, computer and communications engineering (B.Eng. and M.Eng by Research from Nanyang Technological University, Singapore). During her career, she has taken on various advisory and consultancy roles for national level cyber security initiatives. She also participates actively as the lead scientist of collaborative projects with industry partners such as MNCs, SMEs and the government agencies. 